\title{
Central retinal artery occlusion associated with persistent truncus arteriosus and single atrium: a case report
}

\author{
Cheng-wei Lu' ${ }^{1}$ Jun Wang ${ }^{2}$, Dan-dan Zhou ${ }^{3 *}$, Ji-long Hao ${ }^{1 *}$, Ling-ling Liang ${ }^{1}$, Xiao-hong Li ${ }^{1}$ and Peng Hui ${ }^{1}$
}

\begin{abstract}
Background: Central retinal artery occlusion (CRAO) is an ocular emergency and most of the cases present with painless sudden persistent loss of vision in the range of counting fingers to perception of light. The presentation of CRAO is associated with a variety of medical conditions. We report a rare case of CRAO associated with persistent truncus arteriosus (PTA) and single atrium in a female patient.

Case presentation: A 23-year-old woman was admitted due to sudden painless visual loss in the left eye. On examination visual acuity of light-perception was noted in the left eye with a left relative afferent pupillary defect. Fundoscopic examination revealed retinal ischemic whitening, constriction of the arteriole and venule with segmentation and typical "cherry-red spot" suggesting CRAO. The patient was treated with ocular massage and anterior chamber paracentesis. She was commenced on $150 \mathrm{mg}$ of aspirin and also received hyperbaric oxygen therapy. An echocardiogram revealed PTA and single atrium. A diagnosis of CRAO associated with PTA and single atrium was made.
\end{abstract}

Conclusion: The ophthalmologist should enquire about congenital and acquired cardiac abnormalities in patients with CRAO and consider such abnormalities to be possible sources of emboli.

Keywords: Central retinal artery occlusion, Persistent truncus arteriosus, Single atrium

\section{Background}

Persistent truncus arteriosus (PTA) and single atrium are both rare congenital cardiac syndromes, and their occurrence together is extremely rare. PTA accounts for under $1 \%$ of all congenital heart diseases, and over $80 \%$ of patients succumb to heart failure in infancy [1]. Central retinal artery occlusion (CRAO) is an ocular emergency, and the etiology of it is usually associated with atherosclerotic risk factors and the presence of intravascular (carotid artery, aortic arch) or intracardiac embolic material. At young age, CRAO may be a manifestation of inherited or acquired thrombophilia [2]. However, CRAO associated with congenital cardiac anomaly is uncommon. According to a literature review,

\footnotetext{
*Correspondence: zhoudan0928@sohu.com; 289736582@qq.com

${ }^{3}$ Department of Radiology, the First Hospital of Jilin University, No. 71 of xinmin St, Changchun, Jilin Province 130021, China

'Department of Ophthalmology, the First Hospital of Jilin University, No. 71

of xinmin St, Changchun, Jilin Province 130021, China

Full list of author information is available at the end of the article
}

a CRAO case with PTA and single atrium has not been described previously. Herein we first report a rare case of CRAO in a Chinese female patient who had both PTA and single atrium with survival to age 23.

\section{Case presentation}

A 23-year-old woman presented with sudden painless visual loss in the left eye of $100 \mathrm{~min}$. She had been diagnosed with PTA at the age of 1 month. In medical history, she had no ophthalmic problem and had maintained good visual acuity. There was no history of vascular occlusion affecting other organs. On examination visual acuity of $20 / 20$ in the right and light-perception was noted in the left eye with a left relative afferent pupillary defect. Anterior segment examination was unremarkable with normal intraocular pressures. Fundoscopic examination revealed retinal ischemic whitening, constriction of the arteriole and venule with segmentation and typical "cherry-red spot" suggesting CRAO (Fig. 1). The patient was treated with ocular massage 


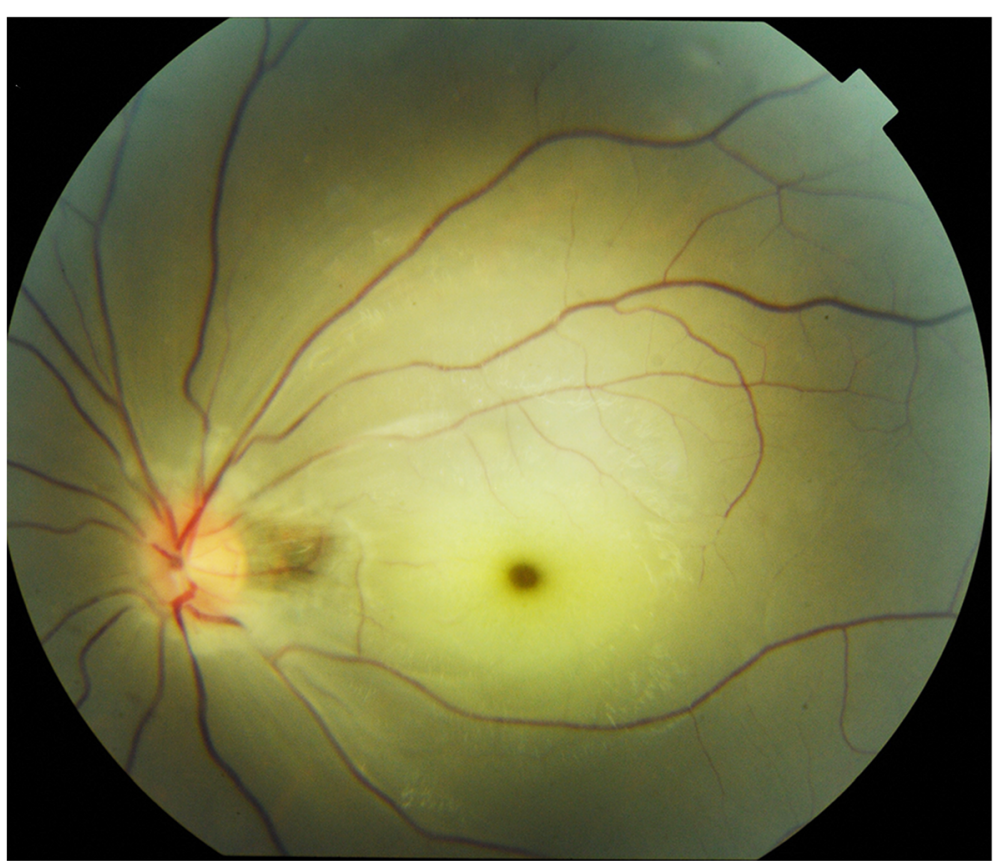

Fig. 1 Fundus photography of the affected eye. Fundus examination revealed retinal chemic whitening, and a cherry-red spot in the foveal area

and anterior chamber paracentesis. She was commenced on $150 \mathrm{mg}$ of aspirin and also received hyperbaric oxygen therapy. Her visual acuity returned to hand motion in the temporal direction. Fluorescein angiography of the right eye carried out on the second day was normal, whereas the left eye showed a delay in arterial filling and large areas of nonperfusion. An echocardiogram was performed (Figs. 2 and 3) and revealed a single and dilated truncal artery overriding both ventricles, a subtruncal ventricular septal defect (VSD) and absence of pulmonary valve and arteries. A diagnosis of persistent truncus arteriosus was established. The annulus size of common truncus was $41 \mathrm{~mm}$. A single atrium, normal function of the left ventricle, and a hypertrophied right ventricle with severe tricuspid regurgitation were shown. Ultrasonography of the carotid arteries was unremarkable. Further

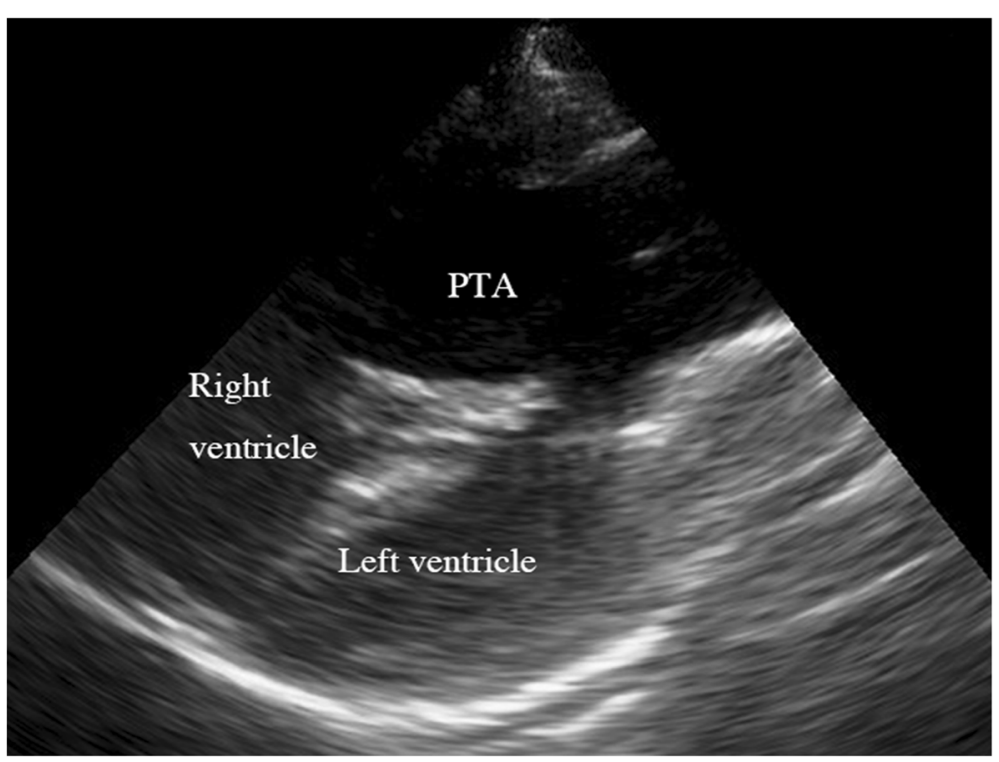

Fig. 2 The image of echocardiogram. An echocardiogram revealed PTA overriding both ventricles 


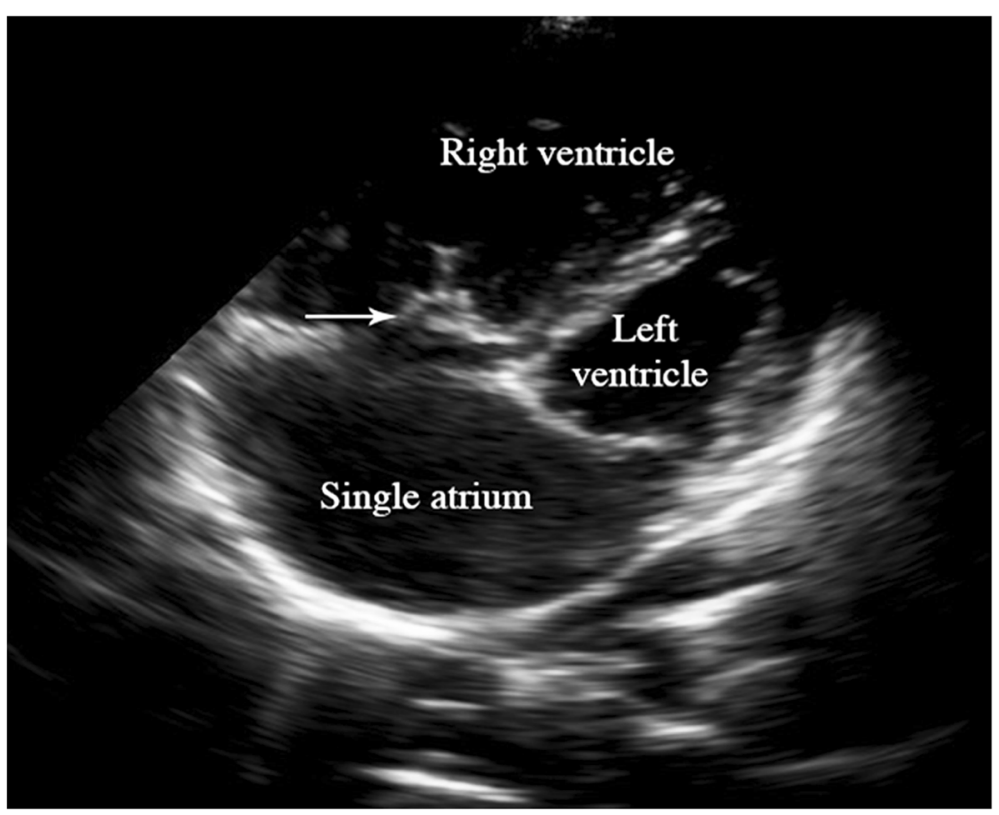

Fig. 3 Echocardiographic image. An echocardiogram revealed single atrium and tricuspid valve prolapse (arrow)

evaluation for an underlying cause was unrevealing, with a normal erythrocyte sedimentation rate, C-reactive protein level, blood count, renal function and ionogram, liver tests, glycosylated hemoglobin and hemostasis. A head computed tomography scan at this time demonstrated no abnormalities.

\section{Discussion}

CRAO is an ocular emergency and the incidence is estimated to be 1 in 100,000 people [2]. In 1859, von Graefes [3] first described CRAO as an embolic event to the central retina artery in a patient with endocarditis. Most of the cases present with painless sudden persistent loss of vision in the range of counting fingers to perception of light. Anterior segment evaluation is usually normal except for the presence of an afferent pupillary defect. Initially, fundus may appear relatively normal. Eventually, hypoxia results in ischemic whitening of the retina, most pronounced at the posterior pole. A cherry red spot is typical and found in about $90 \%$ of cases.

The presentation of CRAO in a young individual is associated with a variety of medical conditions, including hyperhomocysteinemia, temporal arteritis, systemic lupus erythematosus, sickle cell disease, platelet aggregation abnormalities, and migraine. But Cardiogenic emboli are a very rare cause of CRAO. While atrial fibrillation and left ventricular dysfunction were shown to be the most common cardiac sources, extracardiac

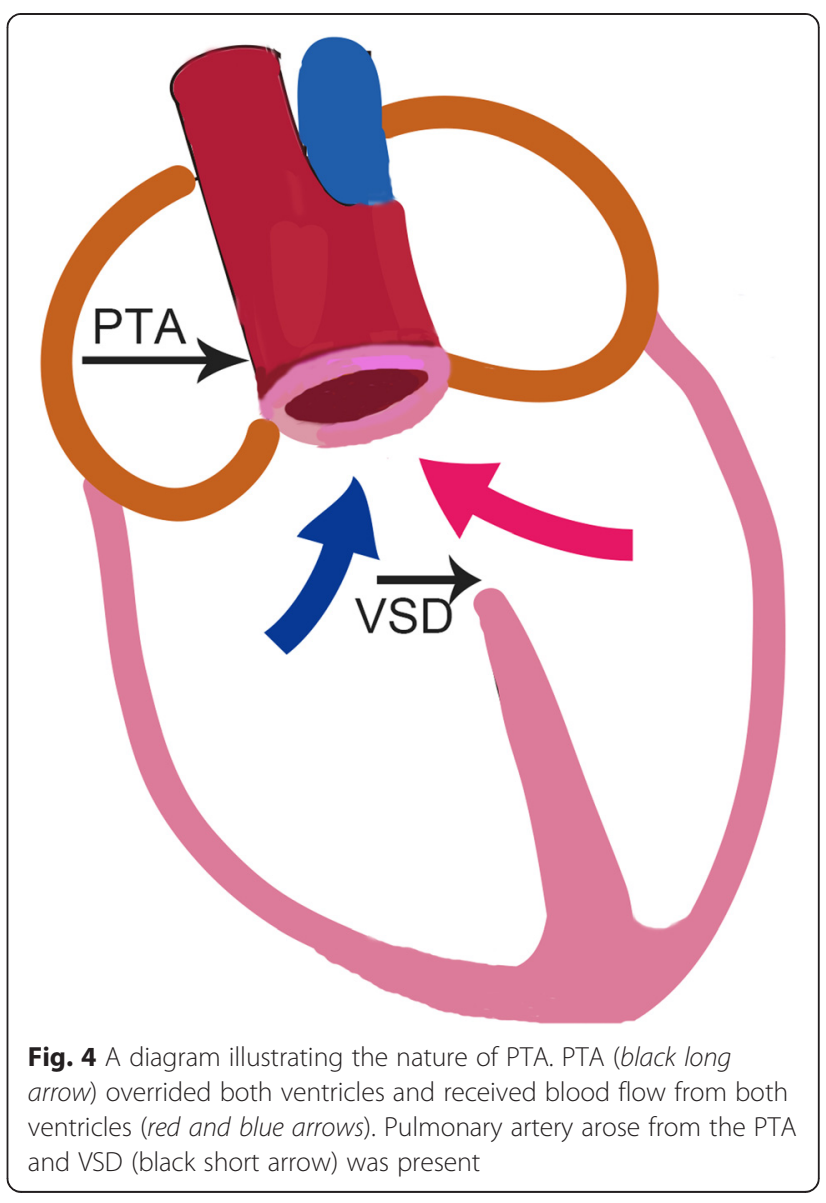


sources (mainly aortic and carotid plaques) are associated with CRAO in the large majority of patients [4]. Unfortunately, the source of embolism remains unclear in about $45 \%$ of the patients [5]. Other proposed mechanisms include infective endocarditis, left atrial thrombosis and myxoma, aortic arch atheroma, mitral annulus calcification, left atrial appendage thrombus, valvular abnormalities, papillary fibroelastoma, and patent foramen ovale [6]. In the present cases, no other source of embolism could be found except for the described exceedingly rare congenital heart disease. However, association of CRAO with PTA and single atrium, to our knowledge, has not been reported.

We first present a patient with CRAO who was born with the simultaneous occurrence of two congenital cardiac defects, which are individually uncommon. PTA is usually termed as a single great artery arising from the base of the heart that supplies systemic, coronary and pulmonary blood flow (Fig. 4). Almost all cases are associated with VSD. The natural history of PTA is poor. Although this patient did not have well-developed pulmonary arteries (PAs), sufficient circulation to the PAs by means of collateral vessels and balanced systemic circulation may have been present. This probably resulted from protection of the pulmonary circulation induced by the brachial artery or hypoplastic branch PAs in this PTA patient. Single atrium is a failure of development of the embryologic components that contribute to the atrial septal complex. Both PTA and single atrium can lead to lower-than-normal oxygen levels in the arterial blood, pulmonary hypertension, and secondary tricuspid valve prolapse.

Therapy for CRAO remains highly uncertain and controversial. It is thought that removal of the embolus within 90 min gives the best chance at recovery, but irreversible damage occurs after $4 \mathrm{~h}$ [7]. Treatment measures include increasing blood oxygen content and dilation of retinal arteries, reducing intraocular pressure immediately through medications (acetazolamide $500 \mathrm{mg}$ i.v or orally, topical beta blocker), ocular massage, and anterior chamber paracentesis, and the use of thrombolytics [8]. Hayreh and Zimmerman [9] described that visual outcomes differed between types of CRAO. Vision improves in $22 \%$ of eyes with nonarteritic CRAO, whereas it improves in $67 \%$ of eyes with nonarteritic CRAO with cilioretinal artery sparing. In the present case of nonarteritic CRAO including cilioretinal artery, visual improvement appeared to be poor. These findings suggested that earlier intervention is necessary for the management of CRAO to preserve visual function.

\section{Conclusion}

In summary, we first describe an extremely rare case of CRAO associated with PTA and single atrium. When we encounter acute painless visual impairment, CRAO should be considered and treated as soon as possible, and the ophthalmologist should enquire about congenital and acquired cardiac abnormalities in patients with CRAO and consider such abnormalities to be possible sources of emboli.

\section{Consent}

Written informed consent was obtained from the patient for publication of this case report and any accompanying images. A copy of the written consent is available for review by the Editor of this journal.

\section{Abbreviations \\ CRAO: Central retinal artery occlusion; PTA: Persistent truncus arteriosus; PAs: Pulmonary arteries; VSD: Ventricular septal defect.}

\section{Competing interests}

The authors declare that they have no competing interests.

\section{Authors' contributions}

CWL and DDZ drafted the manuscript. JW, LL, XHL and PH reviewed the literature. JLH revised the manuscript. All authors read and approved the final manuscript.

\section{Acknowledgements}

The authors don't have any acknowledgement regarding this case report.

\section{Author details}

'Department of Ophthalmology, the First Hospital of Jilin University, No. 71 of xinmin St, Changchun, Jilin Province 130021, China. ${ }^{2}$ Department of Ultrasound, the First Hospital of Jilin University, No. 71 of xinmin St, Changchun, Jilin Province 130021, China. ${ }^{3}$ Department of Radiology, the First Hospital of Jilin University, No. 71 of xinmin St, Changchun, Jilin Province 130021, China.

Received: 18 August 2015 Accepted: 10 October 2015

Published online: 19 October 2015

\section{References}

1. Slavik Z, Keeton BR, Salmon AP, Sutherland GR, Fong LV, Monro JL, Persistent truncus arteriosus operated during infancy: long-term follow-up. Pediatr Cardiol. 1994;15:112-5.

2. Leavitt JA, Larson TA, Hodge DO, Gullerud RE. The incidence of central retinal artery occlusion in Olmsted County, Minnesota. Am J Ophthalmol. 2011;152:820-3.

3. von Graefes A. Ueber Embolie der Arteria centralis retinae als Ursache plotzlicher Erblindung. Arch Ophthalmol. 1859;5:136-57.

4. Mouradian M, Wijman CA, Tomasian D, Davidoff R, Koleini B, Babikian VL. Echocardiographic findings of patients with retinal ischemia or embolism. J Neuroimaging. 2002;12:219-23.

5. Babikian V, Wijman CA, Koleini B, Malik SN, Goyal N, Matjucha IC. Retinal ischemia and embolism. Etiologies and outcomes based on a prospective study. Cerebrovasc Dis. 2001;12:108-13.

6. Kramer M, Goldenberg-Cohen N, Shapira Y, Axer-Siegel R, Shmuely H, Adler Y et al. Role of transesophageal echocardiography in the evaluation of patients with retinal artery occlusion. Ophthalmology. 2001;108:1461-4.

7. Hayreh SS, Kolder HE, Weingeist TA. Central retinal artery occlusion and retinal tolerance time. Ophthalmology. 1980;87:75-8.

8. Cugati S, Varma DD, Chen CS, Lee AW. Treatment options for central retinal artery occlusion. Curr Treat Options Neurol. 2013;15:63-77.

9. Hayreh SS, Zimmerman MB. Central retinal artery occlusion: visual out come. Am J Ophthalmol. 2005;140:376-91. 